\title{
Abnormal aortic-to-radial arterial pressure gradients resulting in misdiagnosis of hemodynamic instability
}

\author{
André Denault, MD · Alain Deschamps, MD, PhD
}

Received: 4 February 2009/ Accepted: 17 March 2009/Published online: 30 April 2009

(C) Canadian Anesthesiologists' Society 2009

This Perioperative Cardiovascular Rounds article features a common cause of perceived hemodynamic instability resulting from abnormal aortic-to-radial arterial pressure gradients. The possible mechanisms and the diagnostic criteria are considered. Permission was obtained from the Research Ethics Board of the Montreal Heart Institute to archive this article into a databank for educational purposes and to reproduce the anonymized echocardiographic images for publication.

A 69-year-old patient with no clinical evidence of subclavian steal underwent uneventful open replacement of the mitral and aortic valves. Following the bivalvular replacement, it was difficult to wean the patient from cardiopulmonary bypass (CPB). Despite normal filling pressures and an infusion of noradrenaline $30 \mu \mathrm{g} \cdot \mathrm{min}^{-1}$, the mean arterial pressure at the end of CPB was only $75 \mathrm{mmHg}$. A transesophageal echocardiographic examination was performed to establish the cause of the perceived hemodynamic instability. The examination revealed preserved left and right ventricular systolic function, no valvular abnormalities, and no reason for the apparent hypotension. With assistance from the surgeon, a new cannula was transduced into the aortic root, and a

Electronic supplementary material The online version of this article (doi:10.1007/s12630-009-9088-8) contains supplementary material, which is available to authorized users.

A. Denault, MD $(\varangle) \cdot$ A. Deschamps, MD, PhD

Department of Anesthesiology, Montreal Heart Institute and Université de Montréal, 5000 Belanger Street, Montreal,

QC H1T 1C8, Canada

e-mail: denault@videotron.ca

A. Denault, MD

Division of Critical Care, Centre Hospitalier de l'Université de Montréal, Montreal, QC, Canada large aortic-to-radial arterial pressure gradient was identified. The radial artery pressure was $86 / 77 / 69 \mathrm{mmHg}$ (systolic, mean, diastolic) while the aortic root pressure was $132 / 107 / 92 \mathrm{mmHg}$ (Fig. 1). In light of the $46 \mathrm{mmHg}$ systolic pressure gradient, the patient was rapidly weaned from vasoactive support using the aortic root pressure as a guide, followed by easy separation from CPB.

This scenario can be commonly observed in the cardiac operating room (Fig. 1) and in the intensive care unit (Fig. 2, Supplementary Video \#1) in patients who are thought to be hemodynamically unstable. Despite previous descriptions of these observations, ${ }^{1,2}$ the mechanisms responsible for this abnormal pressure gradient remain poorly understood ${ }^{3}$ and its occurrence is not routinely recognized. The pressure gradient between the aortic root and the radial artery is normally $<20 \mathrm{mmHg}$, being higher in the distal arteries. ${ }^{4}$ In our clinical experience, an aortic root-to-radial artery systolic pressure gradient $>25 \mathrm{mmHg}$ occurs in approximately $30-50 \%$ of cardiac procedures. Such pressure gradients typically manifest during the

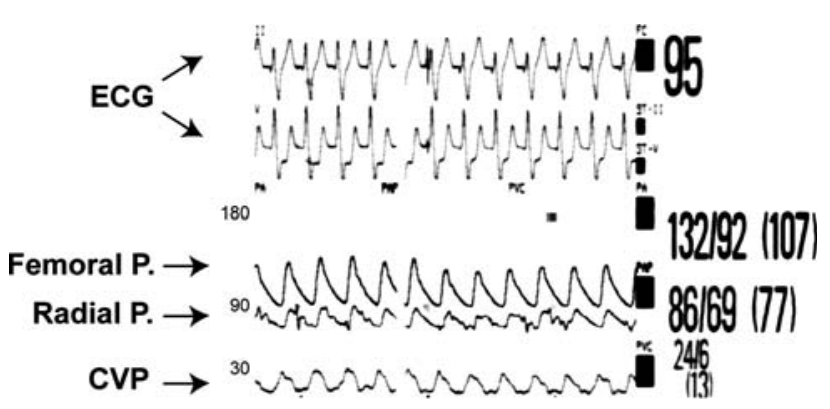

Fig. 1 Electrocardiographic and hemodynamic traces from a patient undergoing mitral and aortic valve surgery. An abnormally large pressure gradient occurred at the conclusion of the valve repair and was observed between the femoral and radial arteries. ECG electrocardiogram; $P$ pressure; $C V P$ central venous pressure 


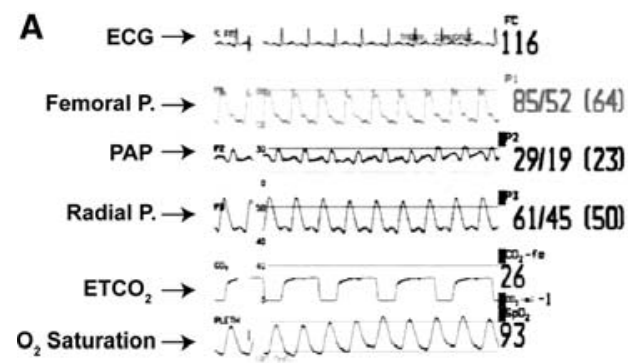

C

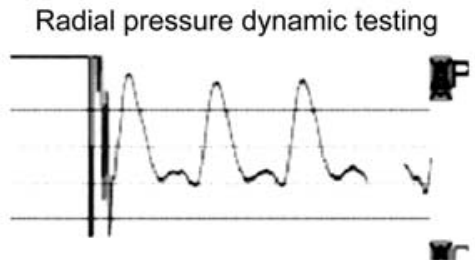

Fig. 2 a Electrocardiographic and hemodynamic traces of a patient in septic shock. An abnormally large femoral-radial arterial pressure gradient was observed. b The gradient is clearly observed when combining both pressures on the same scale. $\mathbf{c}$ Normal response during
B

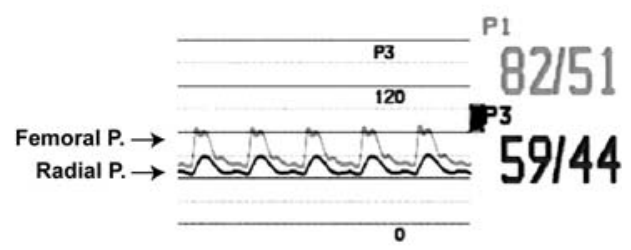

D Femoral pressure dynamic testing

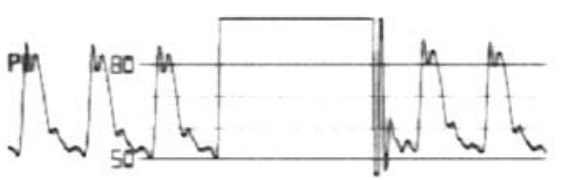

dynamic testing of the radial artery transducer. d Normal response during dynamic testing of the femoral artery transducer. $E C G$ electrocardiogram; $P$ pressure; $P A P$ pulmonary artery pressure $E^{2} \mathrm{CO}_{2}$ end-tidal carbon dioxide; $\mathrm{O}_{2}$ oxygen (Supplementary Video \#1)
A

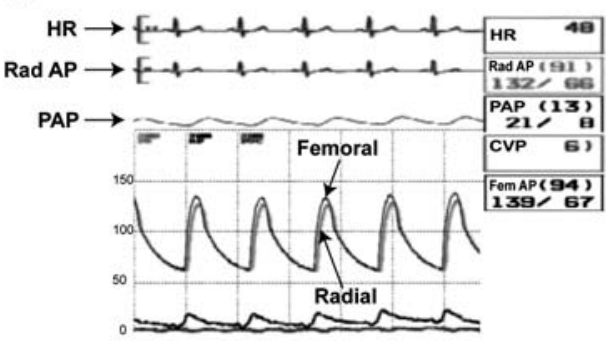

C

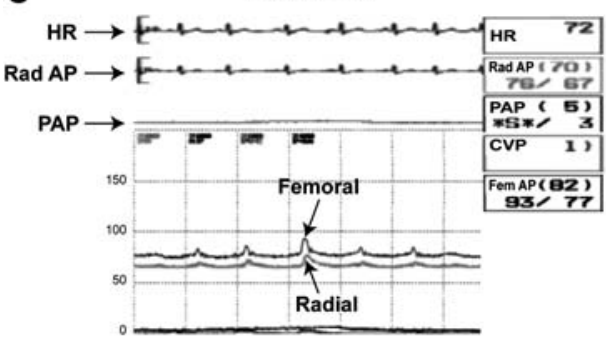

Fig. 3 Radial-to-femoral artery pressure gradient during cardiac surgery. a Before cardiopulmonary bypass (CPB), a normal gradient between the radial (red) and femoral (white) arteries was observed. b During the early part of CPB, no abnormality in gradient was observed. c The gradient appears at the beginning of CPB. d After

course of CPB. Maximum pressure gradients are usually observed just after separation from CPB (Fig. 3, Supplementary Video \#2). In some patients, these gradients resolve towards the end of the procedure; however, there is limited predictability regarding their dynamic variability with respect to time. Due to the difficulty in predicting
B Early CPB

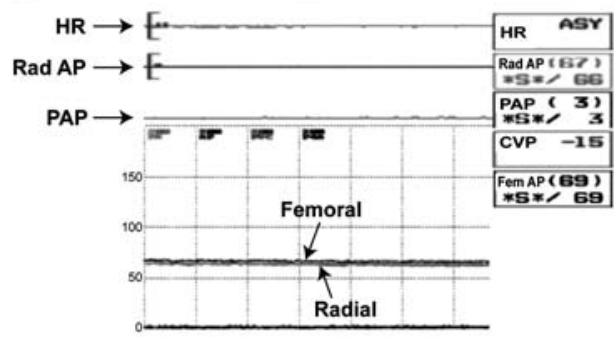

D

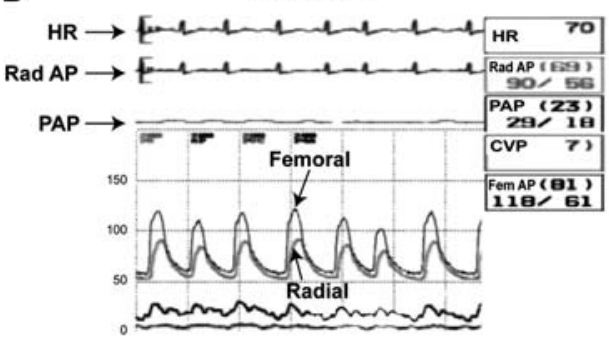

$\mathrm{CPB}$, the systolic and mean femoral artery pressures were $118 \mathrm{mmHg}$ and $81 \mathrm{mmHg}$, respectively. The systolic and mean radial artery pressures were $90 \mathrm{mmHg}$ and $69 \mathrm{mmHg}$, respectively. $H R$ heart rate; $A P$ arterial pressure; $P A P$ pulmonary artery pressure; Rad radial artery; Fem femoral artery; $C V P$ central venous pressure

elevated aortic-to-radial arterial pressure gradients, our routine practice is to monitor both radial and femoral arterial pressures in all patients undergoing cardiac surgery. The femoral line serves as the primary guide for monitoring systemic blood pressure, while the radial line remains the easiest conduit for blood sampling for point-of-care 


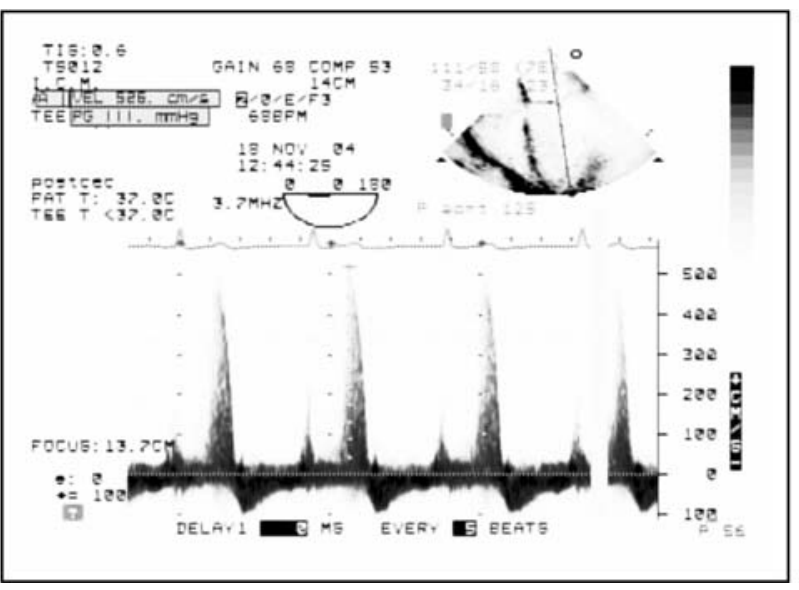

Fig. 4 Step-by-step non-invasive diagnosis of an abnormal radial-tocentral arterial pressure gradient. This can be estimated using continuous wave (CW) Doppler interrogation of the mitral valve. In the presence of mitral regurgitation, a pressure gradient can be calculated across the mitral valve. This gradient represents the difference in systole between the left ventricular (LV) end-systolic pressure (LVESP) and the left atrial pressure (LAP). If the aortic valve is normal, the LVESP is similar to the systolic arterial pressure.

laboratory tests. Using dual arterial lines has the added advantage of permitting blood sampling without causing transient interruptions in the key blood pressure waveform.

Also, we apply an ultrasound-guided approach for inserting the femoral artery catheter before surgery, particularly in patients at increased risk of hemodynamic instability. Transesophageal echocardiography and transthoracic echocardiography can also be used to detect abnormal arterial pressure gradients when clinically significant mitral regurgitation is present. By using continuous wave Doppler interrogation of the mitral valve in the presence of mitral regurgitation, a pressure gradient can be calculated across the mitral valve. This gradient represents the difference between the left ventricular end-systolic pressure (LVESP) and the left atrial pressure (LAP). With a normal aortic valve, the LVESP is similar to the systolic arterial pressure. Consequently, by adding the pressure gradient estimated through Doppler measurement and the LAP estimate using the pulmonary artery occlusion pressure, the LVESP and systolic pressure can be calculated. This pressure should be similar to the radial artery pressure if no gradient is present. Continuous Doppler imaging is used through the mitral valve (Fig. 4). This is the same approach used routinely by cardiologist to estimate the systolic pulmonary artery pressure using the pressure gradient across the tricuspid valve in patients with tricuspid regurgitation. ${ }^{5}$

Early recognition of an abnormally extensive aortic-toradial arterial pressure gradient is the first and most
1- Obtain a CW Doppler signal through the mitral valve

2- Measure the peak gradient

3- The peak gradient $=$ pressure gradient $=L V E S P-L A P=111 \mathrm{mmHg}$

4- LVESP = pressure gradient + LAP

5- The LAP can be estimated with the pulmonary artery wedge pressure ( $v$ wave)

6- LVESP $=111 \mathrm{mmHg}+$ pulmonary artery wedge pressure ( $\mathrm{v}$ wave) $=111 \mathrm{mmHg}+18 \mathrm{mmHg}=129 \mathrm{mmHg}$

7- LV systolic pressure $\approx$ Aortic pressure

8- Aortic pressure measured by surgeon $=125 \mathrm{mmHg}$

9- Radial pressure $=111 \mathrm{mmHg}$ therefore a $14 \mathrm{mmHg}$ gradient is present

Consequently, by adding the pressure gradient estimated through Doppler measurement and the LAP estimate using the pulmonary artery occlusion pressure, the LVESP and systolic pressure can be calculated. In this example, the estimated LVESP, which should be an approximation of the aortic pressure, was much higher than the radial pressure. Therefore, an abnormally large gradient was present. This relationship is valid only in the absence of aortic valve stenosiseither valvular, subvalvular, or supravalvular

important step in excluding intraoperative hemodynamic instability as a cause of persistent hypotension in cardiac surgery. A differential diagnosis of peripheral to central pressure gradients includes: (1) unilateral or bilateral vascular disease; (2) over or under-damping of the transducer signal; (3) inadequate zeroing of transducers; (4) a defective transducer; and (5) idiopathic causes, such as in cardiac surgery and in hemodynamically unstable patients.

Conflicts of interest None declared.

\section{References}

1. Mohr R, Lavee J, Goor DA. Inaccuracy of radial artery pressure measurement after cardiac operations. J Thorac Cardiovasc Surg 1987; 94: 286-90.

2. Dorman T, Breslow MJ, Lipsett PA, et al. Radial artery pressure monitoring underestimates central arterial pressure during vasopressor therapy in critically ill surgical patients. Crit Care Med 1998; 26: 1646-9.

3. Thrush DN, Steighner ML, Rasanen J, Vijayanagar R. Blood pressure after cardiopulmonary bypass: which technique is accurate? J Cardiothorac Vasc Anesth 1994; 8: 269-72.

4. Guyton AC. Textbook of medical physiology. Philadelphia: Saunders; 1981. p. 223.

5. Denault AY, Couture P, Buithieu J, Tardif JC. Transesophageal echocardiography multimedia manual: a perioperative transdisciplinary approach. Marcel Dekker; 2005. 